lich zu Unrecht - oftmals bereits von einem „Ende der Nachbarschaft“ (Brech 2002: 25) als Folge einer extremen Individualisierung gesprochen. Es konnte festgestellt werden, dass sich das Bedürfnis nach nachbarschaftlichen Kontakten sehr ambivalent gestaltet. Einerseits messen die Menschen den Nachbarschaftskontakten im Kontext der Wohnzufriedenheit eine erhebliche Bedeutung bei, andererseits sind die individuellen Bemühungen zur Herstellung von nachbarschaftlichen Sozialkontakten oftmals gering (Reppé \& Reiter 1997).

Empirische Befunde für Wien belegten, dass Nachbarschaftskontakte nach wie vor in einem hohen Ausmaß als wichtig eingestuft werden. ${ }^{4}$ Das oben zitierte ,Ende der Nachbarschaft" ist also nicht auf eine grundsätzliche Geringschätzung von Nachbarschaftskontakten auf Seiten der Großstadtbewohner zurückzuführen. Distanzverhalten und Kontaktbedürfnisse schließen einander nicht aus, eine gewisse Reserviertheit im nachbarlichen Beziehungsfeld ist keineswegs als Wunsch nach Isolation zu interpretieren (vgl. Barre et al. 1977: 146; Schulz 1978: 22).

Die mediale Tagesberichterstattung dazu war sehr einseitig. Sie wies in erster Linie auf das Konfliktpotential ${ }^{5}$ des ethnisch gemischten Wohnens hin, welches bestenfalls als ein Nebeneinander, aber nicht als ein Miteinander zu interpretieren sei. Vor allem den Wiener Gemeindebauten wurden erhebliche Spannungen zwischen Inländern und Neoösterreichern attestiert ${ }^{6}$. Von einer künftigen Verstärkung dieser Konflikte in Wien war angesichts des Faktums, dass es sich bei einem Drittel bis zur Hälfte der Vorgemerkten für wiedervermietete Wiener Gemeindewohnungen um inzwischen eingebürgerte Zuwanderer handelt ${ }^{7}$, ebenfalls die Rede. Vieles an diesen Konfliktszenarien war allerdings bloße Spekulation. Wissenschaftlich fundierte Ergebnisse waren bis zum Erhebungszeitpunkt für Wien nur in einem sehr beschränkten Ausmaß vorhanden.

\title{
2 Problemstellung und Projektziele
}

Im Rahmen des vorliegenden Projekts haben wir versucht, die Nachbarschaftskontakte und -konflikte in verschiedenen Wohnbaukategorien aufzuzeigen und zu interpretieren. Im Mittelpunkt stand dabei die Analyse subjektiver Wahrnehmungen. Der State of the Art der Nachbarschaftsforschung dokumentiert, dass dieser Themen-

4 Immerhin 70\% der inländischen Respondenten in einer 1998 durchgeführten Erhebung bewerteten Nachbarschaftsbeziehungen als wichtig bzw. sehr wichtig. Nur eine Minorität von $4,4 \%$ betrachtete diese als überflüssig (vgl. Kohlbacher 2000).

5 Vgl. Besser Wohnen 11, Oktober 1992: $18 \mathrm{f}$.

$6 \quad$ Vgl. Menasse (1996) und Tanzer (1996).

7 Der Standard, 10.11.1998: 9. 
bereich von verschiedenen wissenschaftlichen Disziplinen mit eigenständigen Forschungsfragen und Schwerpunktsetzungen bearbeitet wird. Nicht selten werden hierbei interdisziplinäre und multidimensionale Ansätze verfolgt und weiterentwickelt. Nach einer kritischen Sichtung des relativ begrenzten vorhandenen theoretischen und empirischen Materials wählten wir unsere Fragestellungen und eine entsprechende Forschungsstrategie. Den Ausgangspunkt bildete nicht ein geschlossener Begriff von „Nachbarschaft", sondern es ist der Versuch unternommen worden, sich an den unterschiedlichen individuellen Definitionen unserer Respondenten zu orientieren.

Wir haben unsere Arbeit auf raumwissenschaftliche, ethnologische und soziologische Theorien und Methoden gestützt. Hinsichtlich der Untersuchung der konkreten Interaktionen schienen uns die Vorgehensweisen der Ethnologie und der Soziologie am geeignetsten. Die Wahl der sozialen Vernetzungen in der Nachbarschaft als Forschungssujet erforderte eine kontextbezogene Analyse, die auch den weiteren Bereich des Wohnens in einem umfassenderen Aufgriff berücksichtigte.

Von dem Faktum ausgehend, dass die sozialräumlichen Rahmenbedingungen und die Mechanismen gemischtethnischen Zusammenlebens im Wohnbereich und deren Konsequenzen für nachbarschaftliche Interaktionen noch unzureichend erforscht waren, versuchte die Studie des ISR diese Forschungslücke für drei konkrete bauliche Bestandskategorien und ihre spezifischen sozialräumlichen Milieus in Wien zu schließen. Hierbei sollten nicht bloß die Nachbarschaftsbeziehungen zwischen „Altösterreichern" und Migranten, sondern vor allem auch die Interaktionen zwischen Migranten unterschiedlicher ethnonationaler Herkunft und Zugehörigkeit ${ }^{8}$ untereinander untersucht werden.

Ein unmittelbarer Zusammenhang zwischen nachbarschaftlichen Interaktionen und baulichen Formen war in diversen einschlägigen Studien empirisch nur sehr bedingt nachzuweisen (vgl. Brech 2003a: 43), dies bedeutet aber nicht, dass die Berücksichtigung baulicher Faktoren, welche nachbarschaftliche Beziehungen im Rahmen der gemeinsamen Nutzung von Hausinfrastruktur begünstigen/belasten können, völlig irrelevant wäre (Mühlich 1978). Verschiedene Raum-, Kontext- und Umgebungsmerkmale machen bestimmte Verhaltens- und Reaktionsweisen der Einheimischen wie auch der Migranten unterschiedlich wahrscheinlich und häufig. Aus diesem Grund wurde auch der baulich-räumliche Aspekt in die umfangreichen Analysen im Rahmen der Projektfragestellungen einbezogen.

Die Wohnintegration von Menschen mit Migrationshintergrund erfolgt in jeweils bestimmten Wohnungsbestandskategorien und in einem konkreten baulich-räumlichen Kontext, dessen Struktur auch Einfluss auf die Häufigkeit, die Art und den Ablauf

8 Ausländische Vergleichsstudien (etwa jene von Arend 2000) fokussierten stets auf eine Analyse der Nachbarschaftsbeziehungen zwischen Migranten einerseits und „Alteingesessenen“/Inländern etc. und klammerten die vielschichtige Problematik des Zusammenlebens sehr heterogener „Migrantennachbarschaften“ weitgehend aus. 
sozialer Kontakte nimmt und somit das interethnische Zusammenleben im Wohnbereich beeinflusst. An raumbezogenen Parametern auf der räumlichen Mikroebene spielen die divergierende infrastrukturelle Ausstattung (WC in- oder außerhalb des Wohnungsverbands, Vorhandensein von Kellerabteilen, Waschküchen etc.) von Wohnhäusern unterschiedlichen Baualters, aber auch das unmittelbare außerhäusliche Wohnumfeld (Grün- und Freiflächen, Sitzgelegenheiten im Freien, Kinderspielplätze) sowie das Ausmaß segregativer Prozesse eine Rolle. Dem gründerzeitlichen Wohnbaubestand wird hierbei mitunter in besonderem Ausmaß ein integratives Potential zugeschrieben, denn die soziale und ethnische Durchmischung auf der Mikroebene der Wohnhäuser soll eine spezifische, sogar integrationsfördernde Atmosphäre kreieren. ${ }^{9}$ Andererseits wird aber auch auf eine Häufung von mit der Zuwanderung einhergehenden Wohnproblemkonstellationen in der gründerzeitlichen Bausubstanz hingewiesen. ${ }^{10}$

Drei Baubestandskategorien, denen der Status jeweils unterschiedlicher sozialräumlicher Milieus zugeschrieben wurde und die in Wien höhere Anteile von Wohnbevölkerung mit Migrationshintergrund aufweisen, wurden einer vergleichenden Analyse unterzogen:

1) der gründerzeitliche Mietwohnungsbestand,

2) Gemeindewohnungen sowie

3) Eigentums- und Genossenschaftswohnanlagen.

Die Qualität des nachbarschaftlichen Zusammenlebens wurde aus der Perspektive von zwei sozialen Bezugsgruppen analysiert, wobei sich zweitgenannte in zwei Subgruppen untergliedert:

- aus derjenigen von Inländern („Altösterreichern“) einerseits

- und aus jener von Personen mit Migrationshintergrund (Subgruppen der ausländischen Staatsbürger und der „Neoösterreicher").

Auf der Basis der Befragung sollten vor allem die folgenden zentralen Fragen beantwortet werden:

- Was ist das Typische der interethnischen Nachbarschaftsbeziehungen?

- Wie und in welchem Ausmaß finden nachbarschaftliche Interaktionen, aber auch Konflikte zwischen Österreichern und Migranten überhaupt statt?

- Welche sind die häufigsten Konfliktursachen?

- Welche Unterschiede bestehen zwischen den drei untersuchten Wohnungsbestandskategorien?

9 Lichtenberger (1990: 176) wies besonders auf das Phänomen der ,,räumlichen ethnischen Unterschichtung “ hin, indem vor allem die bei Inländern häufig unbeliebten Erdgeschoßwohnungen der Gründerzeithäuser in erster Linie von Familien mit Migrationshintergrund bezogen werden.

10 Vgl. dazu Feigelfeld \& Hartig (2001) in Brech (2003b). 